\title{
Treatment of patellar instability in a case of hereditary onycho-osteodysplasia (nail-patella syndrome) with medial patellofemoral ligament reconstruction: A case report
}

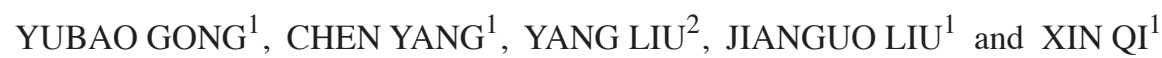 \\ Departments of ${ }^{1}$ Bone and Joint Surgery, and ${ }^{2}$ Radiology, The First Hospital of Jilin University, \\ Changchun, Jilin 130021, P.R. China
}

Received December 9, 2014; Accepted February 8, 2016

DOI: $10.3892 / \mathrm{etm} .2016 .3180$

\begin{abstract}
Hereditary onycho-osteodysplasia, also known as nail-patella syndrome (NPS), is a rare genetic disorder that is primarily characterized by poorly developed nails and patella. Patients with NPS frequently suffer from patellar instability that requires surgical management. The present case report describes a 25-year-old man with NPS. The patient presented with left knee pain and was found to have recurrent left patellar dislocation. The knee pain was first reported 1-year after a minor knee trauma incident. Following complete evaluation, a diagnosis of NPS was reached. The patient underwent surgical intervention using medial patellofemoral ligament (MPFL) reconstruction with a gracilis tendon autograft looped through two transverse 3.2-mm drill holes in the patella and fixed at the natural MPFL insertion site on the medial femoral condyle with an interference screw. The surgery resulted in stabilization of the patella in the femoral trochlea and the patient did not have any subsequent dislocations or subluxations. The patient had an excellent range of knee movement in the follow-up period. This case indicates that MPFL reconstruction in patients with patellar dislocation secondary to NPS can successfully restore normal patellar tracking and result in good range of movement and functional activity.
\end{abstract}

\section{Introduction}

Nail-patella syndrome (NPS), also known as hereditary onycho-osteodysplasia, Turner-Kieser syndrome, Fong disease and iliac horn disease, is a rare disorder involving multiple organs of both ectodermal and mesodermal origin. The

Correspondence to: Dr Xin Qi, Department of Bone and Joint Surgery, The First Hospital of Jilin University, 71 Xinmin Street, Changchun, Jilin 130021, P.R. China

E-mail:imokol@hotmail.com

Key words: medial patellofemoral ligament reconstruction, nail-patellar syndrome, hereditary onchoosteodysplasia, patellar subluxation, dislocation prevalence of NPS is $1 / 500,000$, and there is a high degree of penetrance with variable expression (1). NPS has autosomal dominant inheritance, and a loss of function mutation in the LIM homeobox transcription factor 1- $\beta$ (LMX1B) gene localized in the long arm of chromosome 9 (9q34), has been identified as a genetic abnormality causing NPS $(2,3)$.

A variety of symptoms are associated with this condition, involving, for example, the kidneys, eyes, bone, tendons, ligaments and muscles. Clinical manifestations are extremely variable in frequency and severity and there is inter- and also intra-familial variability $(4,5)$.

NPS primarily affects bones and joints, with patellar involvement in $~ 90 \%$ of patients; however, complete patellar aplasia is observed in only $20 \%$ of cases, and more frequently, the patellae are reduced in size and the knee is unstable because of patellar subluxations or dislocations (6).

Patellar luxation in NPS is often caused by patellar aplasia or medial patellofemoral ligament (MPFL) attenuation. Various surgical options, individually and in combination, have been described for the management of the recurrent patellar dislocation in such cases (7-10). The present case report describes a patient with NPS associated with dislocation of the patella surgically treated by medial patellofemoral ligament reconstruction using the method previously described by Christiansen et al (11).

\section{Case report}

A 25-year-old man presented in January 2010 at the First Hospital of Jilin University (Changchun, China) with pain and an inability to fully flex the knee that had persisted for 1 year. The history of the patient included a fall, following which the symptoms started. The patient also reported having a diffuse pain and swelling around the knee, which was aggravated by going up and down stairs. The pain was located anteriorly in the knee and was described as an aching pain with intermittent sharp and severe pain. The patient also reported instability and apprehension while performing flexion activities. Upon clinical examination, the two patella were located at the same height; however, the left patella was smaller than the right. During range of motion testing, it was found that the left patella exhibited maltracking and was dislocated upon flexing the 

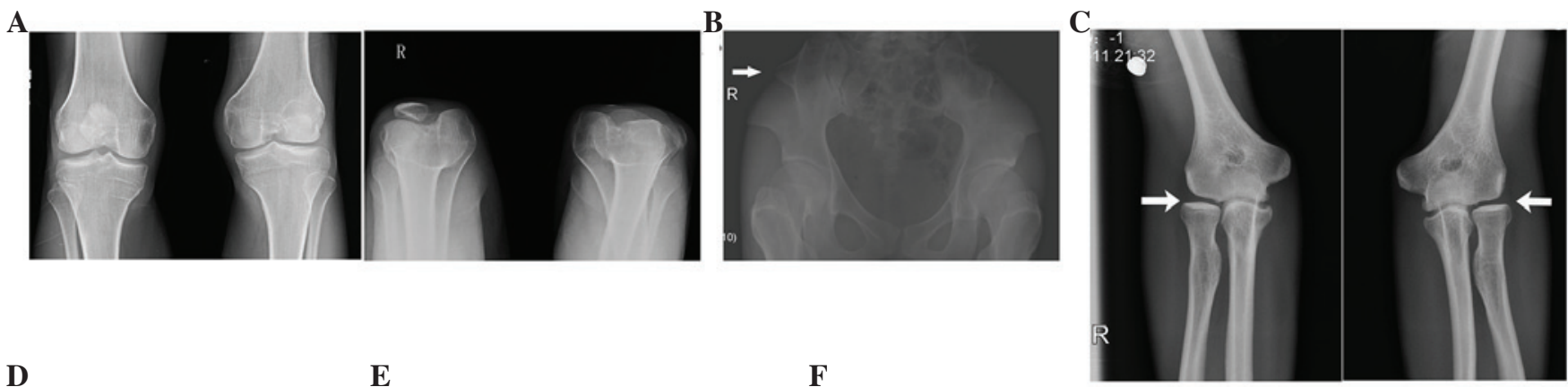

D

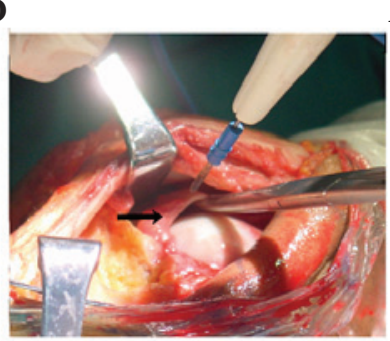

$\mathbf{E}$

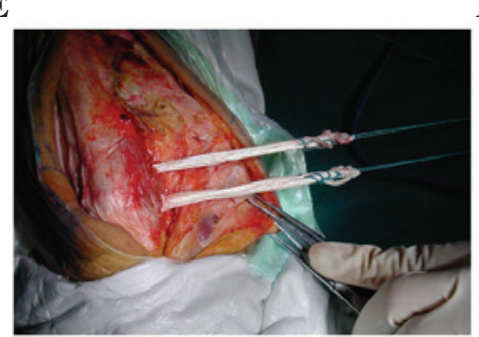

$\mathbf{F}$

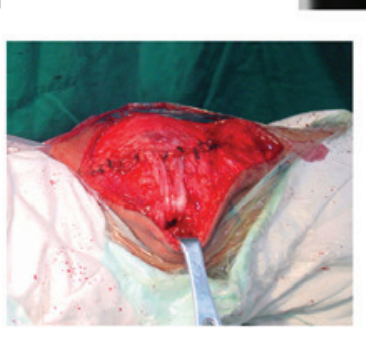

G

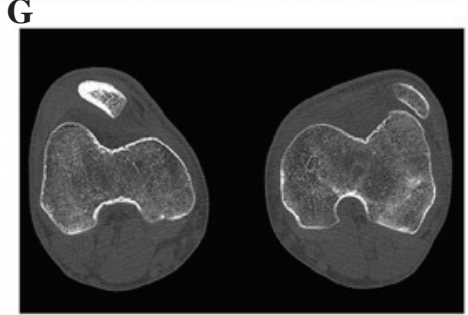

Figure 1. (A) Anteroposterior radiographs revealed a dislocated left patella. Patellar dimensions as measured on the radiographs were $3.33 \mathrm{~cm}$ wide and $3.75 \mathrm{~cm}$ high for the right patella and $2.40 \mathrm{~cm}$ wide and $2.40 \mathrm{~cm}$ high for the left. (B) A right side iliac horn was also found on the pelvic X-ray. (C) Bilateral elbows showed slight subluxation. (D) The medial plicasynovialis was found and resected. (E) The gracilis tendon was harvested through a 3 -cm incision over the pesanserinus. Two transverse 3.2-mm drill holes were made through the patella. (F) The tension and isometricity of the reconstruction was tested through the arc of motion, and once confirmed the tendons were fixed in the femoral condyle with a bioresorbable interference screw. (G) Computed tomography scanning showed that the trochlea was well-developed, but that the left patella was smaller than that on the contralateral side.

knee beyond $5^{\circ}$. The Q-angle measured at $30^{\circ}$ of knee flexion was $5^{\circ}$ in the right knee and $0^{\circ}$ in the left. The patella grind test and apprehension test were negative. The left patella could be manually subluxed to the lateral side without difficulty but could not be manually subluxed to the medial side, indicating lateral structure tightness. Thigh circumferences proximal to the patella were $31.2 \mathrm{~mm}$ on the right side and $30.2 \mathrm{~mm}$ on the left, indicating quadriceps atrophy on the left side. The left patellar tilt test was negative and no palpable tenderness was identified around either knee. There were no signs of hyperlaxity in other joints. The fingernails were normal, but the toenails were relatively poorly developed.

Anteroposterior radiographs (Advantx, GE Healthcare Life Sciences, Chalfont, UK) revealed a dislocated left patella. The dimensions of the patellae as measured on the radiographs were $3.33 \mathrm{~cm}$ in width and $3.75 \mathrm{~cm}$ in height for the right patella and $2.40 \mathrm{~cm}$ in width and $2.40 \mathrm{~cm}$ in height for the left (Fig. 1A). A right side iliac horn was also observed (Fig. 1B). Bilateral elbows showed slight subluxation (Fig. 1C).

The medical history of the family was traced and it was found that characteristic symptoms of NPS were exhibited in two generations of the family (Fig. 2).

A corrective procedure was performed through an anterior longitudinal incision $\sim 15 \mathrm{~cm}$ long. The lateral retinaculum was tight and so a retinacular release was performed. The medial plicasynovialis was identified and dissected (Fig. 1D). The medial patellofemoral ligament structure was not found; therefore, the medial patellofemoral ligament was reconstructed using the surgical technique described by Christiansen et al (11). The gracilis tendon was harvested through a $3-\mathrm{cm}$ incision over the pes anserinus. Two transverse 3.2-mm drill holes were made through the patella (Fig. 1E). The adductor tubercle was exposed and

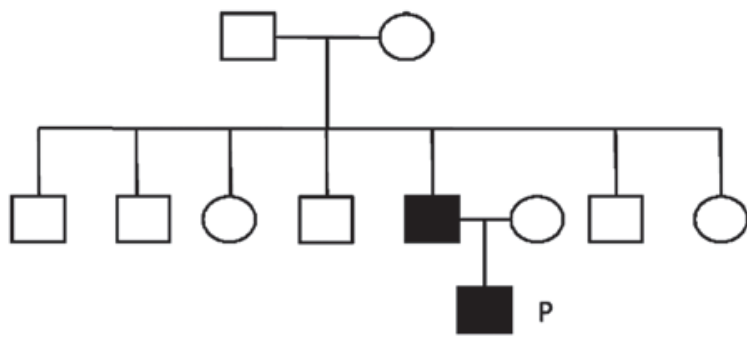

Figure 2. Family members in two generations exhibited characteristic symptoms of nail patella syndrome (NPS). P indicates the patient; black symbols indicate symptoms of NPS. Circles represent females and squares males.

identified at the medial femoral condyle. A 6-mm hole was drilled at the natural MPFL insertion point, just distal to the tubercle. The tendon passed through the two 3.2-mm holes in the patella. On the medial side, the free ends of the tendon were passed under the fascia to the femoral drill hole. The two tendon ends were tightened into the femoral drill hole using a Beath pin pullout technique (11). The tension and isometricity of the reconstruction were tested through the arc of motion, and once confirmed the tendons were fixed in the femoral condyle with a bioresorbable interference screw (Milagro ${ }^{\circledR}$; DePuy Synthes, Raynham, MA, USA; Fig. 1F).

The patient began passive knee exercises using a continuous passive motion machine (HT-B1, Zhejiang Jinhua Huatong Medical Appliance Co., Ltd., Zhejiang, China) 1 week after the surgery. Strengthening and conditioning exercises were initiated simultaneously. Partial weight-bearing exercises on a straight knee began during week 2 following the surgery and continued until week 6 . After 3 months, the patient was permitted to move freely. Patient informed consent was obtained for this study. 


\section{Discussion}

NPS is a rare autosomal-dominant pleiotropic genetic disorder, with a classical clinical tetrad of symptoms, specifically fingernail dysplasia, hypoplastic or absent patella, hypoplasia of the radial head and capitellum and iliac horns. Nail dysplasia and iliac horns cause little or no disability, but abnormalities affecting the elbows and knees can cause symptoms requiring treatment. Involvement of the patella is observed in the majority of cases, which can include patellar absence or hypoplasia with subluxation (12). The majority of cases are asymptomatic and surgical intervention is not necessary. However, congenital permanent dislocation of the patella (CPDP) requires surgical treatment.

The present case report describes a patient with atypical manifestation. The patient did not have congenital patellar subluxation. Instead, the patient reported knee symptoms following a minor trauma. On the basis of the patient's history and clinical examination, it was presumed that, subsequent to the minor trauma, the medial patellofemoral ligament was torn and this resulted in a chronic subluxed and dislocated state. Morphologically, the abnormal patella was smaller than the patella on the unaffected side. This may have contributed to the patellar instability, and, additionally, it increased the technical difficulty of performing a MPFL reconstruction.

There are numerous methods for the treatment of patella subluxation, the selection of which depends on the underlying cause. A partial list of commonly employed methods includes double-strand gracilis autograft (13), combined medial patellofemoral ligament repair and medial patellotibial ligament reconstruction (14), single-strand middle third quadriceps tendon autograft (15), single-strand adductor magnus split tendon transfer (16), and adductor sling (17) and medial collateral ligament sling techniques (18). The aim of these surgical methods used in MPFL is to provide an effective reconstruction without impairment of normal knee function. Autologous grafts of various types, such as semitendinosus, gracilis, quadriceps, semimembranosus tendons and the medial retinacular tissues have been used. Any bone tunnels are required to be just large enough to enable the graft to pass through. When two strands of tendon are used, the superomedial corner of the patella and the junction of the upper and middle thirds of the medial border of the patella (or just distal to it) are the recommended fixation sites (11). The femoral attachment site can be $10 \mathrm{~mm}$ proximal and $2 \mathrm{~mm}$ posterior from the medial femoral epicondyle, or $4 \mathrm{~mm}$ distal and $2 \mathrm{~mm}$ anterior from the adductor tubercle (11). Fluoroscopic imaging of a lateral view of the knee can be used to identify the femoral insertion point $(19,20)$.

In the present case, according to the preoperative computed tomography scan, the trochlea was well developed (Fig. 1G), but the left patella was smaller than that on the contralateral side. The instability of the patella was thus secondary to MPL weakness or absence and lateral retinaculum contracture. Therefore, an anterior longitudinal incision was chosen to address both of these problems. MPL reconstruction was performed using the method described by Christiansen et al (11) using a free gracilis tendon passed through two transverse tunnels in the proximal part of the patella. The reason for selecting this method was the hypothesis that the double band might provide a stronger grip on the abnormally small patella. An interference screw was used for femoral fixation at the adductor tubercle. Due to the small size of the patella, the holes were located far from the cartilage surface to avoid patellar fracture after surgery. It has previously been suggested that it is important not to breach the anterior cortex of the patella, to reduce the risk of fracture (13). In the present case, care was taken to ensure that neither cartilage violation nor any anterior cortical breach occurred.

After the surgery, the patient exhibited a stable patella with good patellar tracking. The range of motion in the immediate postoperative period was also an acceptable $80^{\circ}$. Three months later, the patient could flex the knee to $130^{\circ}$ and ambulate without crutches. No complications such as fracture, wound infection or re-dislocation occurred.

In conclusion, the occurrence of secondary patella dislocation is common in NPS. The most likely cause is an MPFL tear and corrective surgery should be aimed at reconstructing the ligament. The present case indicates that reconstruction using gracilis tendon is a safe and effective method and can be used in NPS cases caused by an MPFL tear.

\section{References}

1. Sweeney E, Fryer A, Mountford R, Green A and McIntosh I: Nail patella syndrome: A review of the phenotype aided by developmental biology. J Med Genet 40: 153-162, 2003.

2. Romero P, Sanhueza F, Lopez P, Reyes L and Herrera L: c.194 $\mathrm{A}>\mathrm{C}(\mathrm{Q} 65 \mathrm{P})$ mutation in the LMX1B gene in patients with nail-patella syndrome associated with glaucoma. Mol Vis 17: 1929-1939, 2011.

3. Witzgall R: How are podocytes affected in nail-patella syndrome? Pediatr Nephrol 23: 1017-1020, 2008.

4. Granata A, Nori G, Ravazzolo R, Marini M, Castellino S, Sicurezza E, Fiore CE and Mignani R: Nail-patella syndrome and renal involvement. Description of three cases and literature review. Clin Nephrol 69: 377-382, 2008.

5. Turner JW: An hereditary arthrodysplasia associated with hereditary dystrophy of the nails. JAMA 100: 882-884, 1933.

6. Choczaj-Kukula, A and Janniger, CK. Nail-patella syndrome. In emedicine: WebMD. http://emedicine.medscape.com/ article/1106294-clinical\#b4. August 22, 2014.

7. Scuderi G, Cuomo F and Scott WN. Lateral release and proximal realignment for patellar subluxation and dislocation. A long-term follow-up. J Bone Joint Surg Am 70: 856-861, 1988.

8. Brown DE, Alexander AH and Lichtman DM. The Elmslie-Trillat procedure: Evaluation in patellar dislocation and subluxation. Am J Sports Med 12: 104-109, 1984.

9. Shelbourne KD, Porter DA and Rozzi W. Use of a modified Elmslie-Trillat procedure to improve abnormal patellar congruence angle. Am J Sports Med 22: 318-323, 1994.

10. Peterson L, Karlsson J and Brittberg M. Patellar instability with recurrent dislocation due to patellofemoral dysplasia. Results after surgical treatment. Bull Hosp Jt Dis Orthop Inst 48: 130-139, 1988.

11. Christiansen SE, Jacobsen BW, Lund B and Lind M: Reconstruction of the medial patellofemoral ligament with gracilis tendon autograft in transverse patellar drill holes. Arthroscopy 24: 82-87, 2008.

12. Brixey AM Jr and Burke RM: Arthro-onychodysplasia; hereditary syndrome involving deformity of head of radius, absence of patellas, posterior iliac spurs, dystrophy of finger nails. Am J Med 8: 738-744, 1950.

13. Lind M, Jakobsen BW, Lund B and Christiansen SE: Reconstruction of the medial patellofemoral ligament for treatment of patellar instability. Acta Orthop 79: 354-360, 2008.

14. Feller JA, Amis AA, Andrish JT, Arendt EA, Erasmus PJ and Powers CM: Surgical biomechanics of the patellofemoral joint. Arthroscopy 23: 542-553, 2007. 
15. Steensen RN, Dopirak RM and Maurus PB. Minimally invasive "crescentic" imbrication of the medial patellofemoral ligament for chronic patellar subluxation. Arthroscopy 21: 371-375, 2005.

16. Sillanpää PJ, Mäenpää HM, Mattila VM, Visuri T and Pihlajamäki H. A mini-invasive adductor magnus tendon transfer technique for medial patellofemoral ligament reconstruction: a technical note. Knee Surg Sports Traumatol Arthrosc 17: 508-512, 2009

17. Ellera Gomes JL. Medial patellofemoral ligament reconstruction for recurrent dislocation of the patella: a preliminary report. Arthroscopy 8: 335-340, 1992.
18. Deie M, Ochi M, Sumen Y, Yasumoto M, Kobayashi K and Kimura H. Reconstruction of the medial patellofemoral ligament for the treatment of habitual or recurrent dislocation of the patella in children. J Bone Joint Surg Br 85: 887-890, 2003.

19. Sillanpää PJ and Arendt E: Reconstruction of the medial patellofemoral ligament using the adductor magnus tendon. Arthroscopy 28: 1749-1750, 2012.

20. Arendt EA. MPFL reconstruction for PF instability. The soft (tissue) approach. Orthop Traumatol Surg Res 95 (Suppl 1): S97-S100, 2009. 